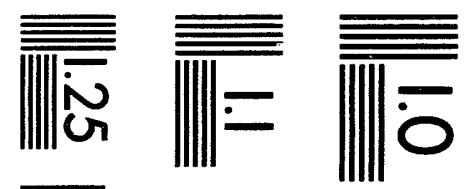

$$
\begin{aligned}
& \text { I⿳亠口冋 }
\end{aligned}
$$

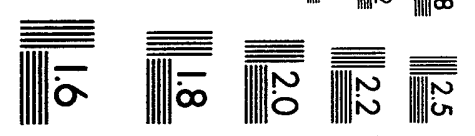



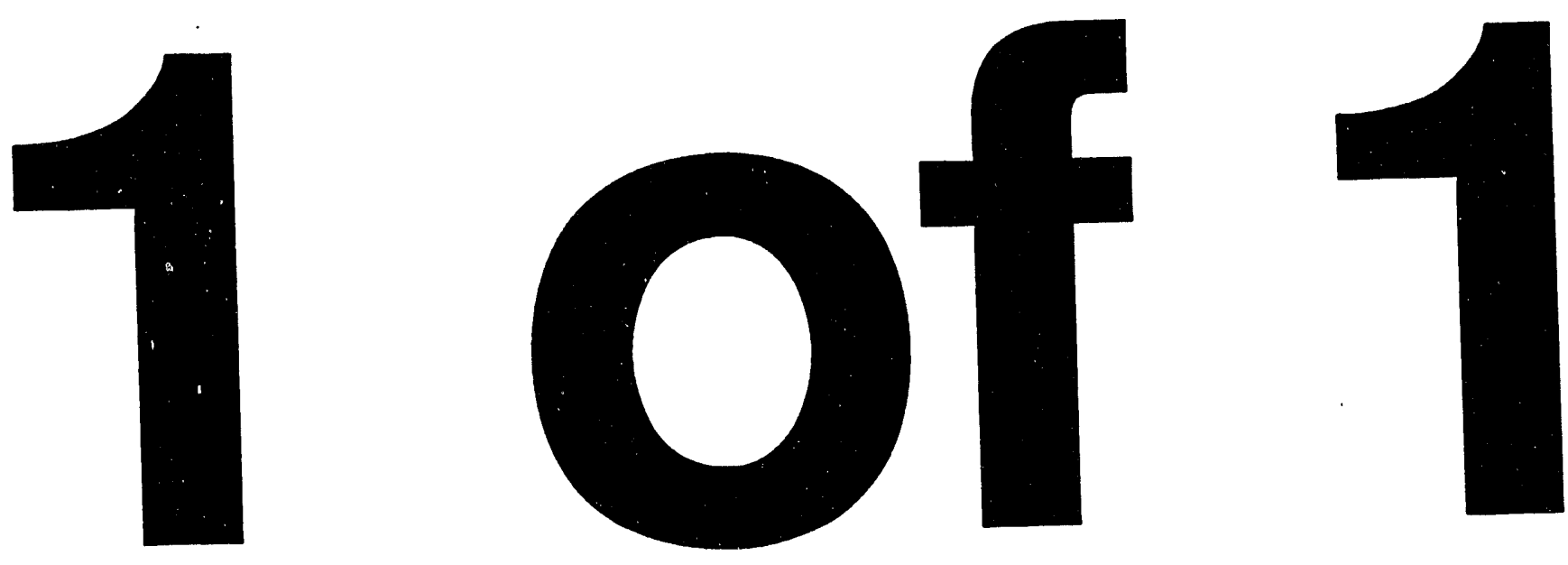
SAND93-3836

Distribution

Unlimited Release

Category UC- 706

Printed April 1994

\title{
Capillary Flow Solderability Test for Printed Wiring Boards
}

F. M. Hosking, F. G. Yost, C. L. Hernandez, and S. J. Sackinger

Materials and Process Sciences Center

Sandia National Laboratories

Albuquerque, NM 87185-0340

\begin{abstract}
This report describes a new technique for evaluating capillary flow solderability on printed circuit boards. The test involves the flow of molten solder from a pad onto different-sized conductor lines. It simulates the spreading dynamics of either plated-through-hole (PTH) or surface mount technology (SMT) soldering. A standard procedure has been developed for the test. Preliminary experiments were conducted and the results demonstrate test feasibility. Test procedures and results are presented in this report.
\end{abstract}




\section{Acknowledgments}

The authors would like to acknowledge the contributions of the many participants on the National Center for Manufacturing Sciences' (NCMS) Printed Wiring Board Interconnect Systems project, especially members from the Surface Finishes Team. Participating companies on the team include AT\&T, IBM, Sandia National Laboratories, Texas Instruments, and United Technologies/Hamilton Standard. Video image analyses were performed by Darrel Frear, SNL/NM. The authors would also like to thank Henry Peebles for his review of the manuscript. This work was performed at Sandia National Laboratories, which is supported by the U.S. Department of Energy under contract number DE-AC04-94AL85000. 


\section{Contents}

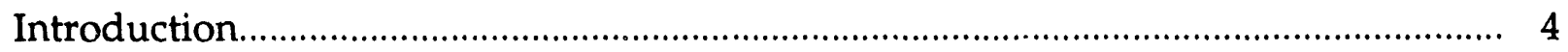

Materials and Capillary Flow Test Procedure................................................................ 5

Preliminary CFTV2 Test Results and Observations................................................... 7

Summary and Conclusions................................................................................... 9

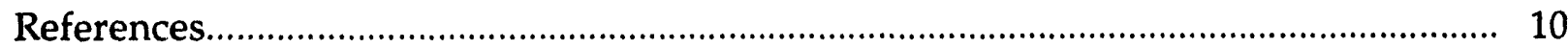

Table:

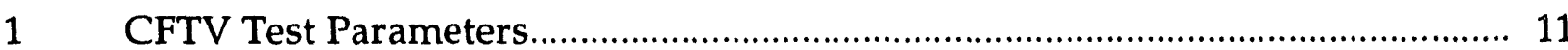

Figures:

1 Capillary flow test geometry for controlling solder wetting. The ratio of line width to pad radius $(\mathrm{l} / \mathrm{r})$ can be varied to control solder spreading from the metal base or pad onto the connected metal strip.

2 The latest version of the capillary flow test vehicle, CFTV2, is shown above.

3 CFTV2 wetted length as a function of temperature and solder paste weight (net solder) for different line widths (pad radius $=0.040$ ).

4 CFTV2 wetted length as a function of temperature and solder pellet weight for different line widths (pad radius $=0.040 "$ ). Samples were immediately solder floated after being coated with an RMA flux

$5 \quad$ CFTV2 wetted length as a function of different line widths (pad radius $=0.040^{\prime \prime}$ ) for $18 \mathrm{mg}$ solder pellets at $260^{\circ} \mathrm{C}$. Samples were solder floated immediately (bottom curve) or 30 minutes (top curve) after flux application.

6 Nominal CFTV2 wetted length after a 30 minute flux predry with $18 \mathrm{mg}$ solder pellets at $245^{\circ} \mathrm{C}$ for different line widths (pad radius $=0.040^{\prime \prime}$ ).

$7 \quad$ Nominal CFTV2 wetted length after a 30 minute flux predry with $18 \mathrm{mg}$ solder pellets at $260^{\circ} \mathrm{C}$ for different line widths (pad radius $=0.040^{\prime \prime}$ ).

8 Nominal CFTV2 wetted length as a function of different line widths (pad radius $=0.040^{\prime \prime}$ ), solder pellet weights, and test temperatures with a 10 minute flux predry (twenty minute predry for the $245^{\circ} \mathrm{C}$ tests)

9 Nominal CFTV2 wetted length as a function of different line widths (pad radius $=0.040^{\prime \prime}$ ), solder pellet weights, and test temperatures with a 30 minute flux predry (twenty minute predry for the $245^{\circ} \mathrm{C}$ tests). 


\section{Introduction}

Existing solder wettability tests ${ }^{1-4}$ (e.g., wetting balance, area-of-spread, meniscus rise, rotary dip, globule) are not clearly understood or uniformly accepted. None of these wettability tests simulate the capillary flow physics of either plated-through-hole (PTH) or surface mount technology (SMT) soldering. PTH and SMT assembly involve solder flow into a small diameter annulus or the gap between a surface mount device and a printed wiring board. Production yield statistics and failure rate data, when available, have demonstrated the need for understanding the fundamentals of wetting through testing of simulated production soldering and incorporating the concepts learned from testing into soldering technology. In this study, a new capillary flow solderability test is presented that simulates typical capillary flow and satisfies existing capillary flow models. This new capillary flow technique offers a potential soldering index for evaluating most microelectronics geometries. It can be directly applied to the control of solder flow on a printed wiring board (PWB). Solder flow can be restricted by adjusting the line width to pad radius ratio. This ability to predict capillary flow would significantly improve solder placement and wetting.

Common to the capillary flow test vehicle's geometry is a metal strip extending from a circular base pad (Figure 1). Consider the circular metallization pattern having radius, $r_{C}$, and let a small volume of solder wet and spread to a radius $r<r_{C}$, such that capillary equilibrium occurs at a contact angle, $\theta_{\mathrm{c}}$. Let the solder volume be increased to a value $V_{0}$, just large enough to allow the solder to spread to the metallization radius. Any further increase of volume will increase the contact angle to a value $\theta_{+c}>\theta_{C}$, but will not increase the radius since the waterloo metallization extends only as far as $r_{c}$. In this quadrajunction configuration, there exists an excess pressure that would drive solder flow if more metallization were available. Consider the additional metallization of a very slender rectangular strip of width $\delta<\mathrm{r}_{\mathrm{c}}$ connected to the circular pad. The excess pressure would move solder onto the strip for certain values of the ratio $\delta$ to $r_{c}$. Solder would flow a distance, $x$, reducing the pressure over the circular metallization until it equals that above the strip. To simplify the flow or spreading calculations, it is assumed that the test geometry is small enough to neglect the effect of gravity on solder shape. Thus, the solder on the circular metallization would have a spherical cross section, while the solder on the strip would be circular. After solder flows onto the strip, an equilibrium contact angle, $\theta_{c}$, is established on the circular metallization. Although the molten solder dissolves and reacts with the substrate, the change of solder volume 
during this process is negligible. The pattern can be fabricated with different pad radii, line widths, and line lengths by either etching or depositing additional metal layers. Either single-sided substrates or doubled coupons with specified gaps can be used to simulate various capillary flow conditions. Theoretical analysis suggests that a line width to pad radius ratio $(1 / \mathrm{r})$ of 0.5 or greater is necessary for solder to flow along the conductor line on a single test vehicle. The test procedure and preliminary experimental results are presented below.

\section{Materials and Capillary Flow Test Procedure}

The capillary flow test vehicle (CFTV) used in this study was fabricated using conventional PWB materials and fabrication technologies. The CFTV demonstration substrate, $0.060^{\prime \prime}$ thick, was an epoxy resin laminate reinforced with glass fiber cloth (FR-4). Copper patterns $(0.5 \mathrm{oz}$.) were printed and etched per the specified "lollipop" test geometry. Additional copper was electrodeposited on the vendor $\mathrm{Cu}$ to a final conductor thickness of $35 \mu \mathrm{m}(1 \mathrm{oz}$. Cu). Ninety one snap-out test specimens were available per test panel. Powder-free finger cots or latex gloves were used when handling individual specimens to minimize contamination from oils, greases, salts, or other foreign debris. Transporting of CFTVs was done with stainless steel tweezers by gripping along the CFTV edge.

The CFTV design has evolved into the version, CFTV2, shown in Figure 2. The substrate has duplicate test patterns with line width-to-pad radius ratios $(1 / \mathrm{r})$ of $0.25,0.5,0.75$, and 1.0. The pad radius was held constant at $0.040 "$, with line widths ranging from 0.010 to $0.040 "$. The line length (or maximum possible capillary flow) was $1.5^{\prime \prime}$. One millimeter $(1.0 \mathrm{~mm})$ reference marks were also patterned onto the TVs and can be used as calibration or datum points during video image analysis of the floated coupons. A buss bar and connecting conductive lines between test patterns were also incorporated into the TV design for depositing other surface finishes (e.g., electroless $\mathrm{Cu}, \mathrm{Sn}$, and $\mathrm{Au}$ ). Free surface spread tests were conducted with a single CFTV. Restricted capillary flow between controlled gaps can be evaluated by stacking two TVs together ("doubled" TVs) and maintaining a constant gap with corner shims.

Soldermask and organic solderability preservative (OSP) are other PWB surface features that can be applied to the CFTV. Three CFTV2 sets having a Cu surface finish with a 
benzotriazole-based OSP coating were fabricated for initial testing. The sets differed only in the application of soldermask (none, 0.001 " or 0.003 " thick). Soldermask was applied by liquid photo imaging $(0.001 ")$ or with dry film photoresist $(0.003 ")$. The board vendor applied the OSP coating before shipping to retain solder wettability during subsequent storage and handling.

Preliminary CFTV2 wetting experiments were conducted with eutectic $\mathrm{Sn}-\mathrm{Pb}$ (63Sn$37 \mathrm{~Pb}, \mathrm{wt} . \%$ ) solder paste and pellets. The paste consisted of $85 \%$ solder and the balance rosin mildly activated (RMA) flux and carrier. RMA liquid flux and solder pellets were also applied to the CFTV before testing. The liquid flux was diluted 1:1 with isopropyl alcohol yielding a nominal $25 \%$ solids flux. The CFTV samples were cleaned before solder floating per the following procedure. The baseline method degreases substrates in trichloroethylene, followed by a rinse in isopropyl alcohol. The substrates are then cleaned in a $10 \% \mathrm{HCl}$ and deionized water solution for 3 minutes to remove the vendor applied OSP, rinsed in hot tap water, rinsed in deionized water, rinsed in isopropyl alcohol, and finally blown dry with technical grade nitrogen gas. Since most commercial precleaners are based on proprietary chemistries that typically change and could consequently affect test result repeatability, precleaning standardization is very important. The technique provides a consistent method for preparing uniform $\mathrm{Cu}$ test surfaces.

Test specimens are coated with flux following the precleaning step. TVs are gently agitated in a flux bath for 5 to 10 seconds. After slow withdrawal from the flux, the TVs are held vertically for approximately 15 seconds and blotted along their bottom edges to remove excess flux. After fluxing and draining, solder pellets or paste are placed on each TV pad per the following procedure: (a) solder pellets of known weight are dipped in flux and placed immediately on the fluxed CFTV pads or (b) solder paste is applied to the fluxed CFTVs with a dispensing gun; the dispensed paste is accurately measured ( $\pm 0.1 \mathrm{mg}$ ) by taring out the TV's pre-weight on an analytical balance and then weighing the TV after applying the solder paste.

CFTV preheating was not done during preliminary testing, since typical reflow machine soldering thermal profiles were difficult to reproduce. A flux predry was not considered for the original CFTV test procedure. As tests were conducted, however, a hold period before solder floating was found to significantly improve test consistency by vaporizing 
the alcohol carrier of the flux. The effects of flux drying will be discussed below in more detail.

CFTV tests were conducted by floating samples on a standard thermostatically controlled solder pot. The total surface area of the solder bath was sufficiently large enough to avoid touching the sides of the solder pot. Test temperatures were maintained within $\pm 2{ }^{\circ} \mathrm{C}$. The nominal baseline test temperature was $245^{\circ} \mathrm{C}$. CFTV2s were initially floated for 60 seconds, and subsequently for longer times (90-120 seconds) to capture the complete wetting event. A minimum of 15-25 seconds typically elapsed before the applied solder melted. Samples were carefully removed from the solder bath after testing to minimize agitation of the molten solder on the TV. This was accomplished by holding the TV in a horizontal position until the solder solidified. Flux residues were then removed by rinsing with trichloroethylene.

Capillary flow data were analyzed from recorded video images. A black and white, charged-couple device (CCD) camera and professional video tape recorder with time code generator were used to record the wetting images. The camera was mounted perpendicular to the floated CFTV. A fiber optic light source was used to illuminate the test surface. Digital image analysis was subsequently conducted with a PC-based image processor. Final wetted distance and flow rate for each line width were measured from selected test images. Accept/reject wetting criteria for the CFTV test is still under development. Further work is planned to validate test method repeatability and determine if CFTV test results correlate with PWB assembly results.

\section{Preliminary CFTV2 Test Results and Observations}

Preliminary testing used the general procedures described above. CFTV2 test panels were fabricated by Alternate Circuit Technology, Ward Hill, MA. The Cu surface finishes were coated with Entek Cu-56 OSP. Kester Solder, Des Plaines, Illinois fabricated solder pellets ranging in weight from 4 to $18 \mathrm{mg}$. ESP Solder Plus supplied the solder paste, $85 \%$ 63Sn-37Pb solder with 15\% RMA flux. The TVs were dipped in Alpha 611 RMA flux, nominally $25 \%$ solids. CFTV precleaning was done per the described cleaning procedure. Tests were conducted with both solder pellets and paste. The effects of float temperature and solder volume on capillary flow were investigated. 
The experimental parameters are listed in Table 1. Tests were performed in random order.

CFTV specimens were fluxed and immediately tested without preheating or predrying. The wetting length results are summarized in Figures $3 \& 4$ for the solder paste and pellet tests, respectively. The data represents only a limited number of tests, but does provide some insight into the effects of temperature, solder type and volume, and line width on capillary flow. The wetting distance was generally sensitive to solder type. During reflow, the solder paste typically flowed further than the solder pellets. After closer examination of the test results, it was discovered that 20 to 30 minutes elapsed between fluxing and floating of the solder paste CFTV2 specimens compared to the much shorter times of 5 minutes or less with the solder pellet samples. The longer delay before paste testing was the result of the "tare and weigh" procedure used to determine the paste weight on each test pad. The flux carrier from the TV and solder paste should volatilize more effectively with increasing hold times or a preheat. Less flux splatter and reduced evaporative surface cooling would consequently improve wetting. The extended hold time could also accelerate the chemical reaction between the $\mathrm{Cu}$ finish and flux.

To verify the effect of flux drying time on solder wetting and flow, additional experiments were conducted at $260^{\circ} \mathrm{C}$ with $18 \mathrm{mg}$ solder pellets and a 30 minute hold after fluxing. The effect of drying on wetting is shown in rigure 5. It is clear that hold time has a significant effect on capillary flow.

Preliminary image analyses of videotaped experiments (18 $\mathrm{mg}$ solder pellets and RMA flux) confirmed the sensitivity of solder flow to the predry time. Flux coated samples were allowed to dry for 30 minutes before floating at 245 and $260^{\circ} \mathrm{C}$ for one minute. The results are summarized in Figures 6 and 7. The wetting curves represent the average of both line pattern replicates. The 0.040 " line initially wetted faster than the other line widths. The 0.030 " line, however, eventually gave similar or better capillary flow, as demonstrated by the $245^{\circ} \mathrm{C}$ test. Intermediate wetting rates and lengths were observed and measured on the $0.020^{\prime \prime}$ line pattern. The 0.010 " pattern yielded negligible capillary flow. The results compare quite favorably with the proposed flow model which predicts capillary flow if the $1 / \mathrm{r}$ ratio is $\geq 0.5$ (or line widths $\geq 0.020^{\prime \prime}$ with a pad radius of $0.040 ")$. The wetting rates also appear to be greater at $245^{\circ} \mathrm{C}$ than at $260^{\circ} \mathrm{C}$. 
Oxidation of the Cu surface at the higher temperature could be competing with the wetting/flow mechanism or the reduced activity of the flux.

The video analyses also revealed a problem in the experimental technique. Individual solder pellets on the CFTV2 were observed to melt at slightly different times. A cursory evaluation with a thermocoupled TV revealed that temperature variations of up to $10^{\circ} \mathrm{C}$ are possible during specimen floatation. These temperature variations could influence capillary flow and test repeatability.

Additional experiments were conducted recently to quantify the predry time effects on wetting. Figures 8 and 9 summarize the results. The longer 30 minute predry generally normalized the flow data at each solder volume with increasing test temperature. For example, little difference was observed in the individual 4 and $18 \mathrm{mg}$ solder pellet flow curves as the temperature was increased from 230 to $260^{\circ} \mathrm{C}$. The maximum wetted distance was primarily affected by the solder volume under these test conditions.

The above preliminary results demonstrate the feasibility of the capillary flow solderability test method. The flow results are very sensitive to test and surface conditions. The issues of flux application, CFTV preheating or drying, surface environmental stressing, flow between "doubled" substrates with a controlled gap, and test repeatability are being further investigated. The final goal is to develop a representative test method for determining the solderability of PWBs. The test will be used to evaluate new or modified PWB surface finishes.

\section{Summary and Conclusions}

A new test method was developed to quantify capillary flow solderability on a PWB surface finish. The test is based on solder flow from a pad onto narrow strips or lines. A test procedure and video image analysis technique were developed for conducting the test and evaluating the data. Feasibility tests revealed that the wetted distance was sensitive to the ratio of pad radius to line width $(1 / r)$, solder volume, and flux predry time. Work is underway to verify test repeatability with single floated coupons. The test is capable of characterizing restricted capillary flow between two test vehicles with a controlled gap. 


\section{References}

1. R. J. Klein Wassink, Soldering in Electronics, Electrochemical Publications Limited, Second Edition, 1989, pp. 36-83 and 300-370.

2. C. Lea, A Scientific Guide to Surface Mount Technology, Electrochemical Publications Limited, 1988, pp. 308-377.

3. Solderability Tests for Printed Boards, ANSI-J-STD-003, The Institute for Interconnecting and Packaging Electronic Circuits (IPC) and Electronic Industries Association (EIA), 1992.

4. F. G. Yost, F. M. Hosking, and D. R. Frear (Editors), The Mechanics of Solder Alloy Wetting and Spreading, Van Nostrand Reinhold, 1993. 
Table 1.

\section{CFTV Test Parameters}

\begin{tabular}{|c|c|c|}
\hline Temperature $\left({ }^{\circ} \mathrm{C}\right)$ & Solder Type & Solder Weight (mg) \\
\hline 230 & Pellet & $4.0 \pm 0.1$ \\
\hline 260 & $" \quad "$ & $"$ \\
\hline 230 & $" \quad "$ & $18.5 \pm 0.3$ \\
\hline 260 & $" \quad "$ & $" \quad "$ \\
\hline 245 & $" \quad "$ & $10.3 \pm 0.1$ \\
\hline 230 & Paste* & $4.1 \pm 0.1$ \\
\hline 260 & $" \quad "$ & $" \quad "$ \\
\hline 230 & $"$ & $18.1 \pm 0.1$ \\
\hline 260 & $" \quad "$ & $"$ \\
\hline 245 & $" \quad "$ & $11.0 \pm 0.1$ \\
\hline
\end{tabular}




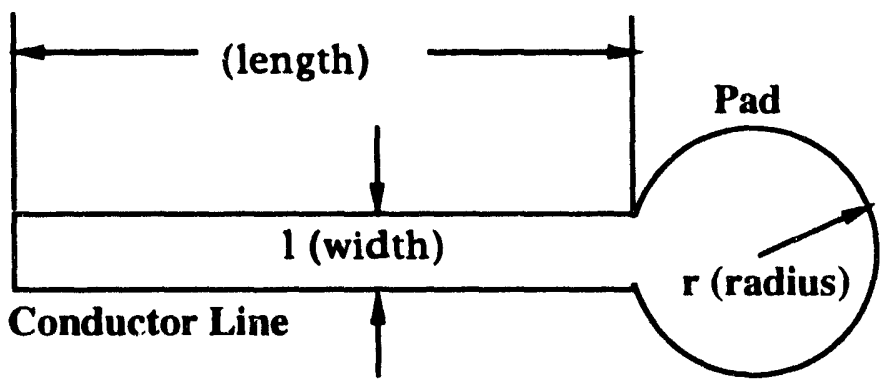

Figure 1. Capillary flow test geometry for controlling solder wetting. The ratio of line width to pad radius $(l / r)$ can be varied to control solder spreading from the metal base or pad onto the connected metal strip.

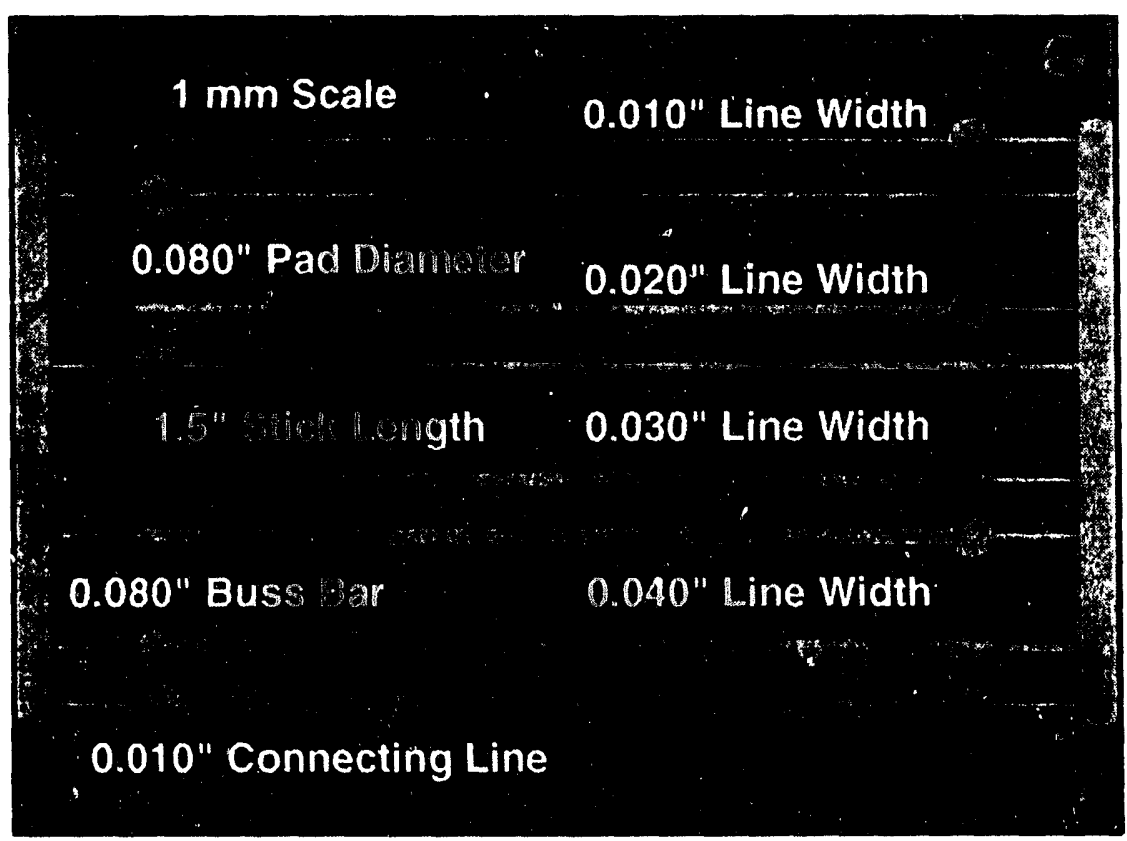

Figure 2. The latest version of the capillary flow test vehicle, CFTV2, is shown above. 


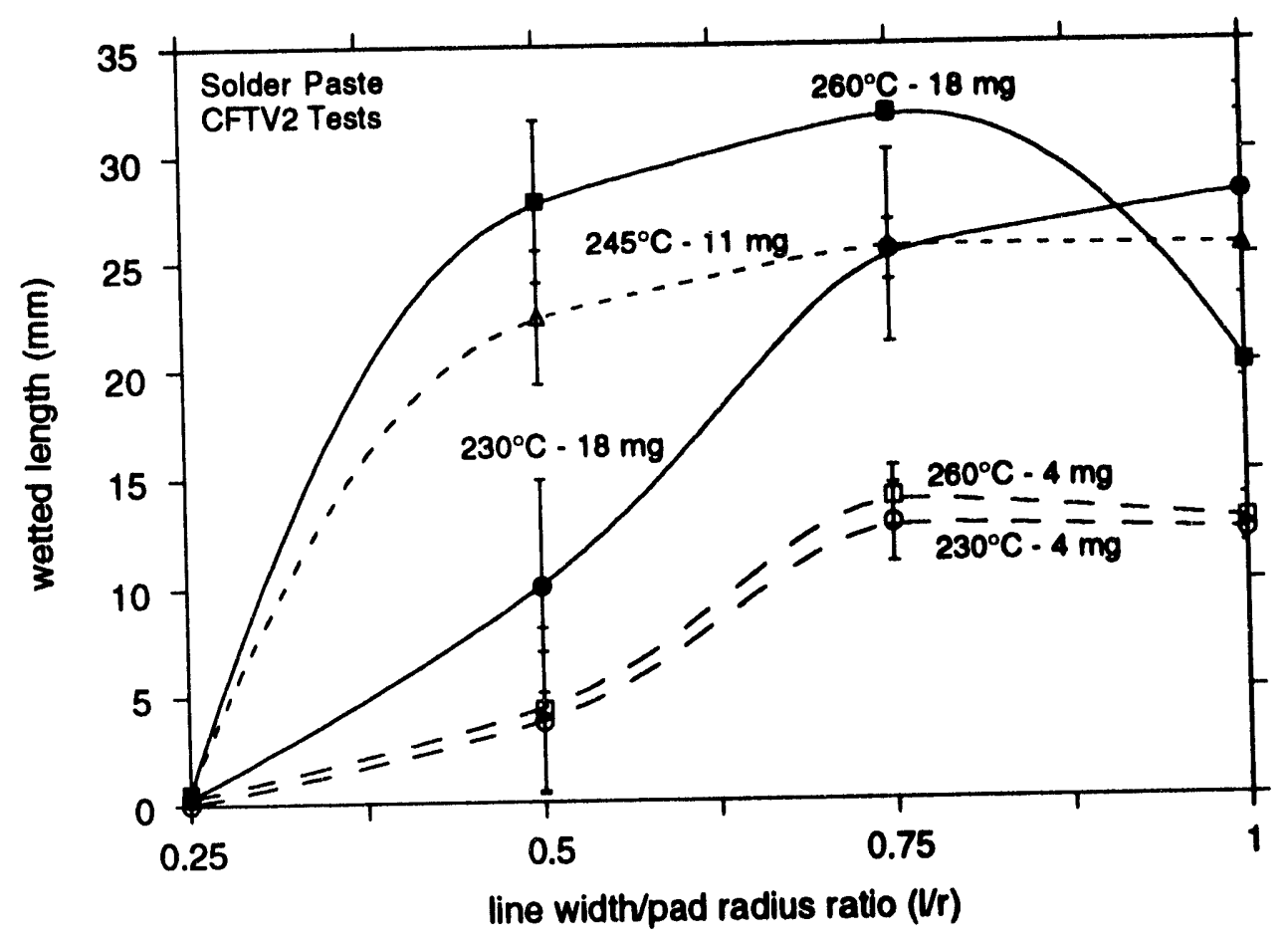

Figure 3. CFTV2 wetted length as a function of temperature and solder paste weight (net solder) for different line widths (pad radius $=0.040 "$ ). 


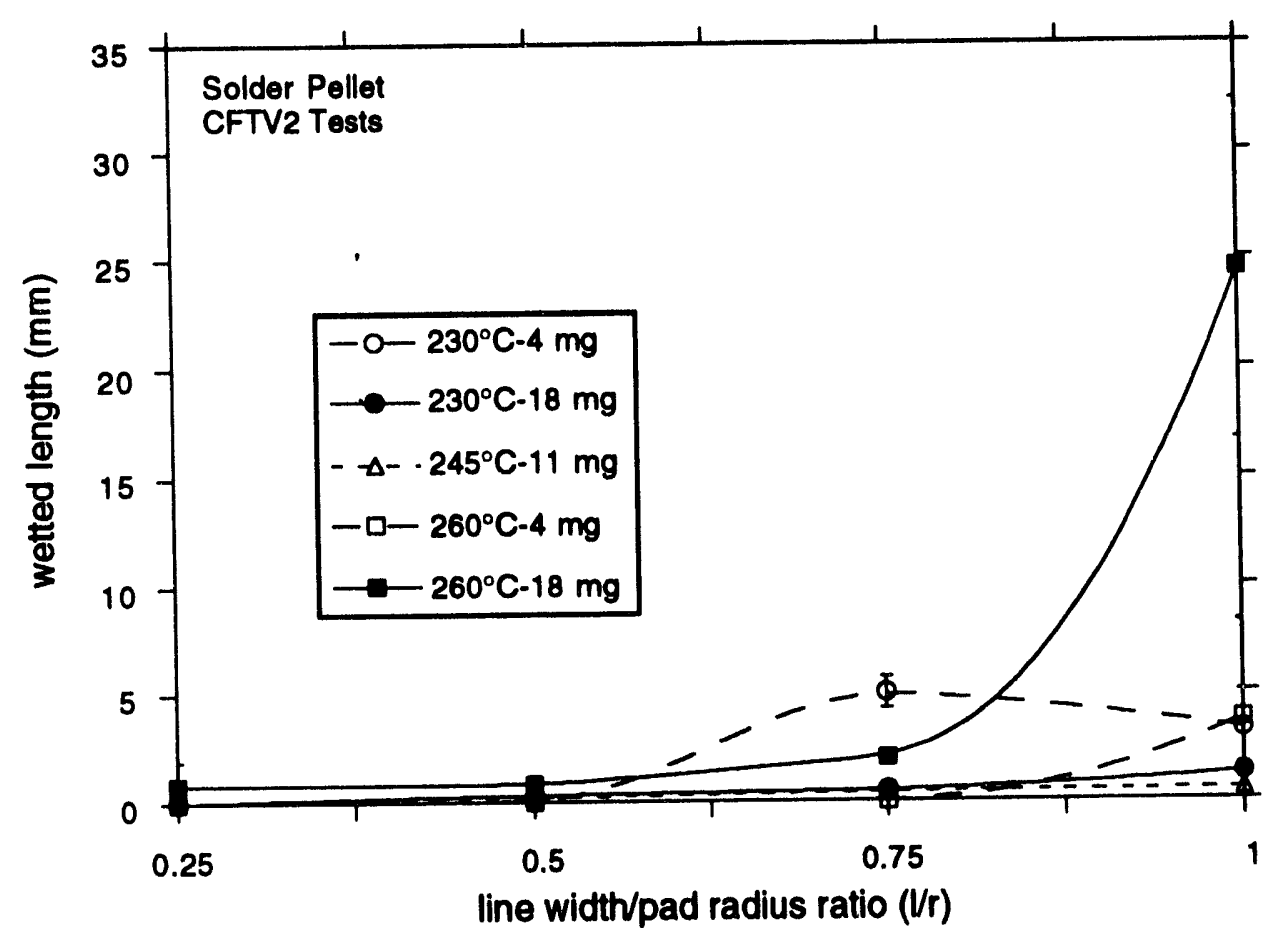

Figure 4. CFTV2 wetted length as a function of temperature and solder pellet weight for different line widths (pad radius $=0.040 "$ ). Samples were immediately solder floated after being coated with an RMA flux. 


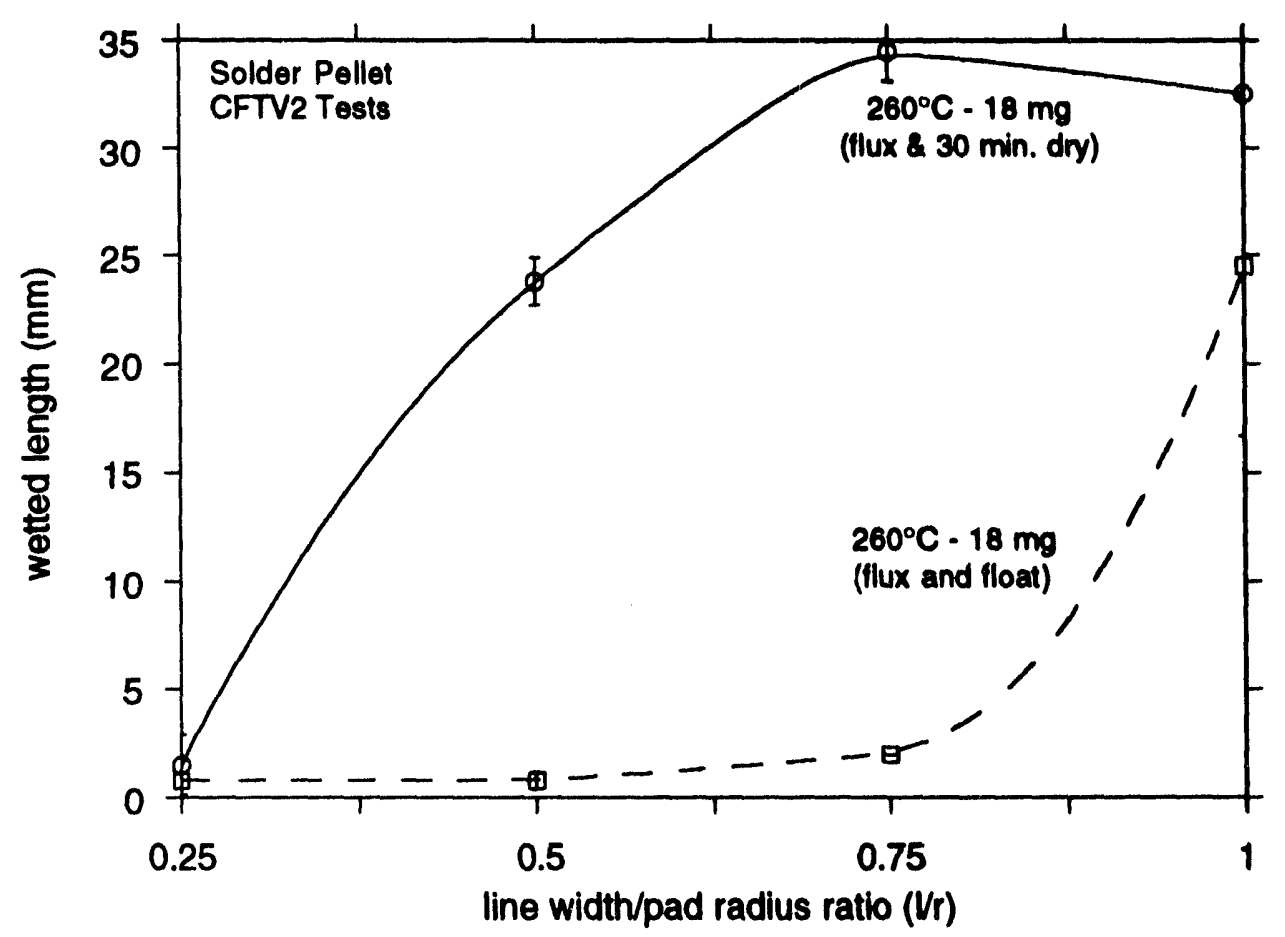

Figure 5. CFTV2 wetted length as a function of different line widths (pad radius = 0.040 ") for $18 \mathrm{mg}$ solder pellets at $260^{\circ} \mathrm{C}$. Samples were solder floated immediately (bottom curve) or 30 minutes (top curve) after flux application. 


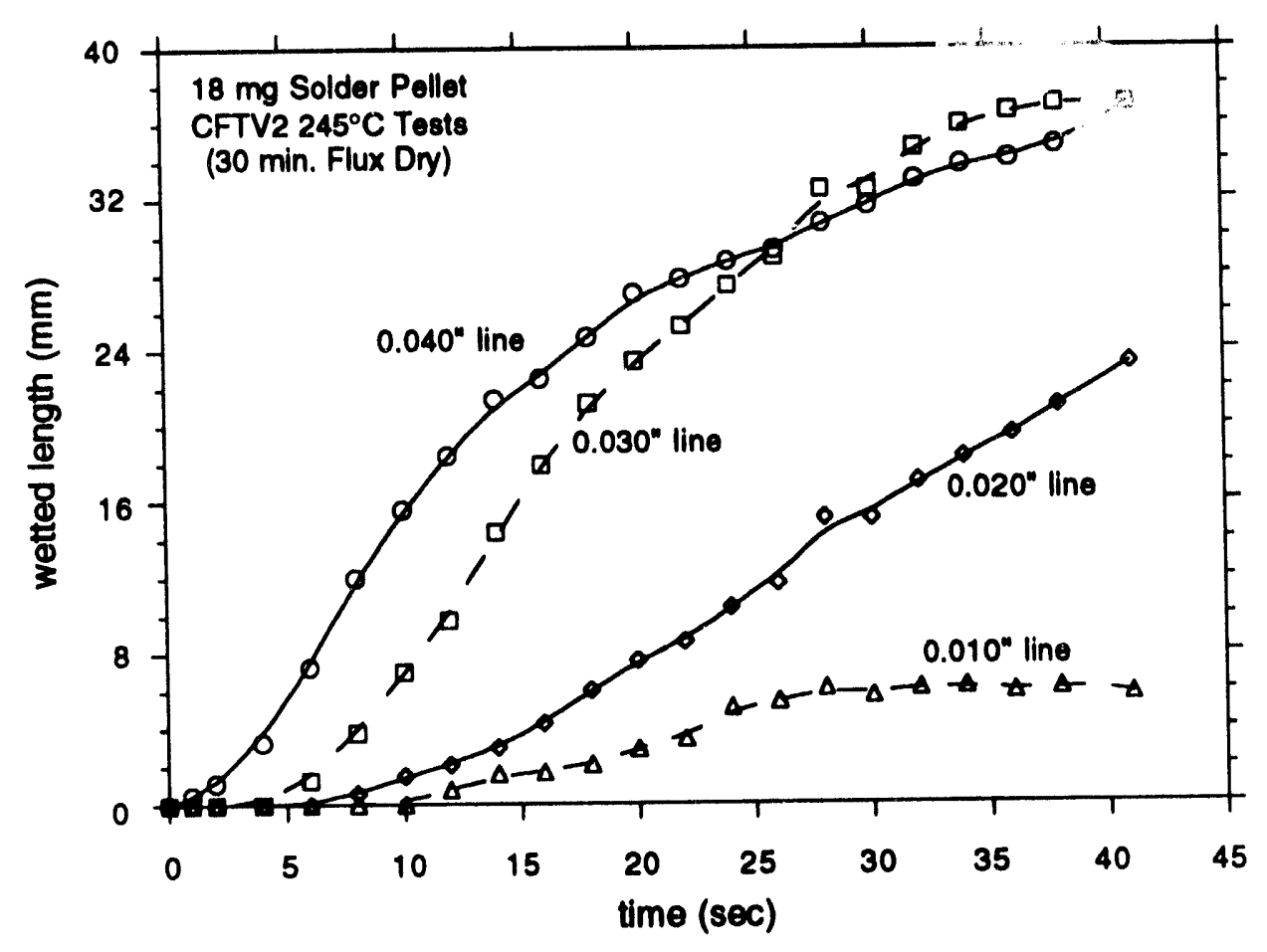

Figure 6. Nominal CFTV2 wetted length after a 30 minute flux predry with $18 \mathrm{mg}$ solder pellets at $245^{\circ} \mathrm{C}$ for different line widths (pad radius $=0.040^{\prime \prime}$ ). 


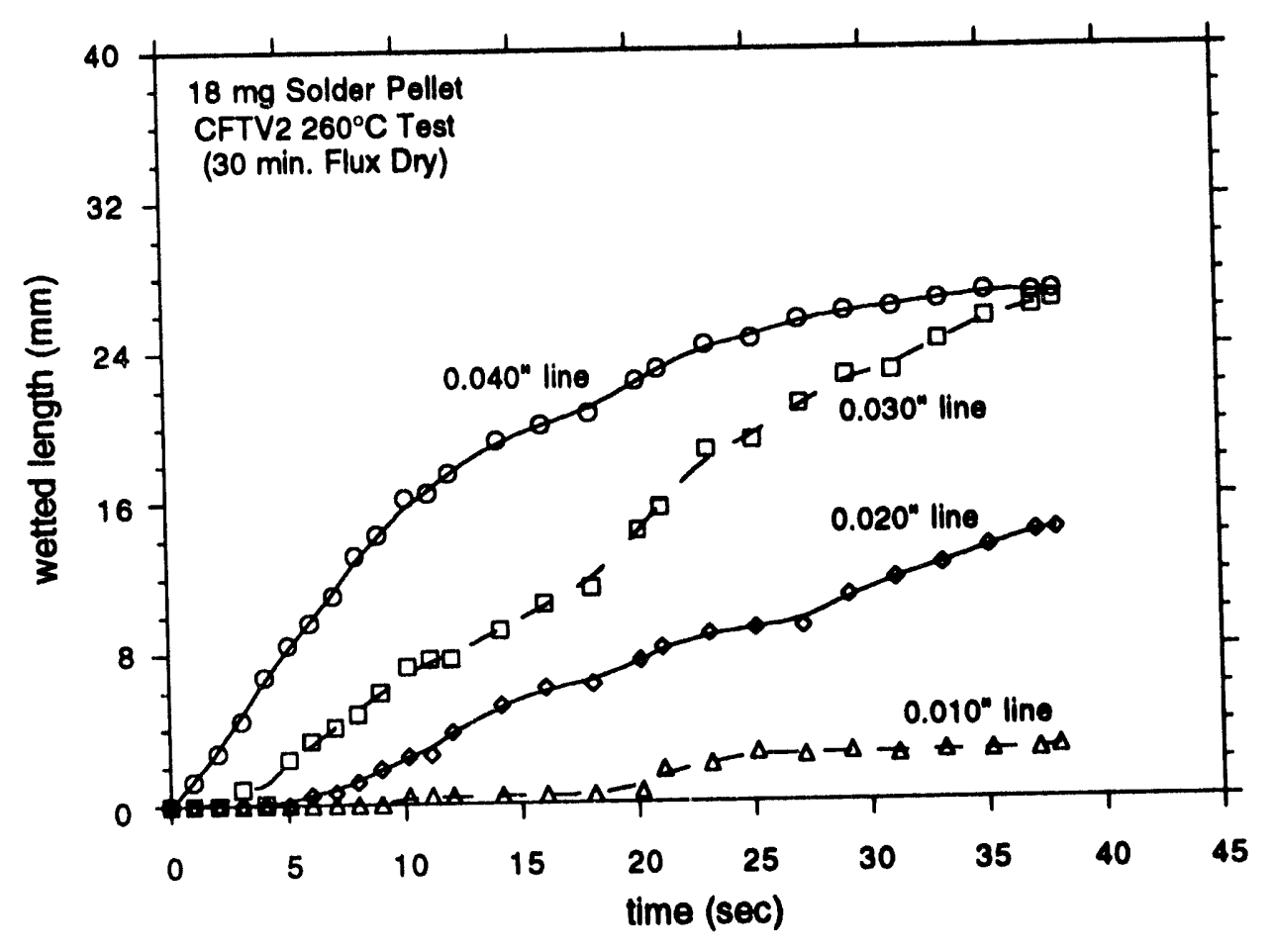

Figure 7. Nominal CFTV2 wetted length after a 30 minute flux predry with $18 \mathrm{mg}$ solder pellets at $260^{\circ} \mathrm{C}$ for different line widths (pad radius $=0.040^{\prime \prime}$ ). 


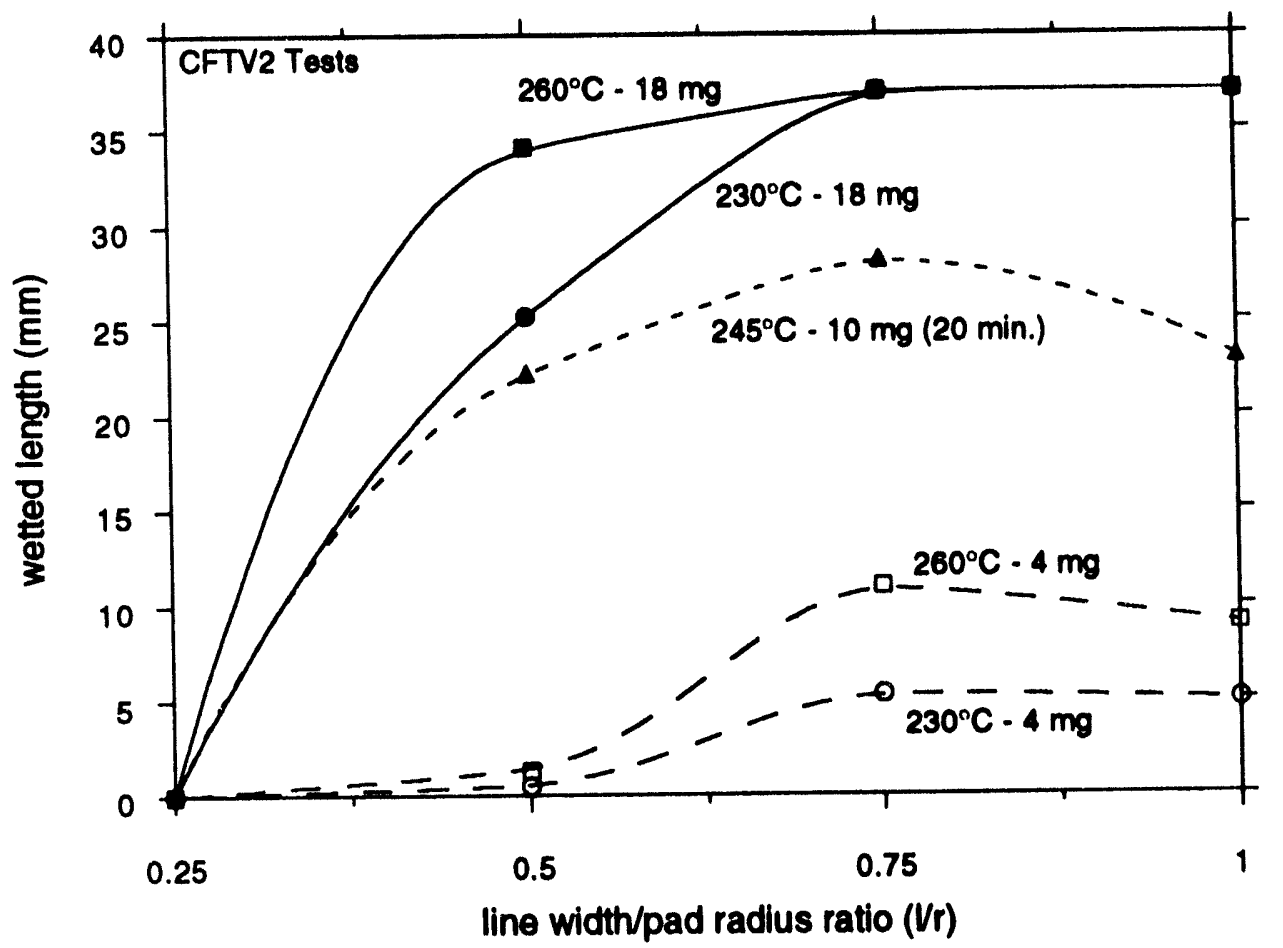

Figure 8. Nominal CFTV2 wetted length as a function of different line widths (pad radius $=0.040 "$ ), solder pellet weights, and test temperatures with a 10 minute flux predry (twenty minute predry for the $245^{\circ} \mathrm{C}$ tests). 


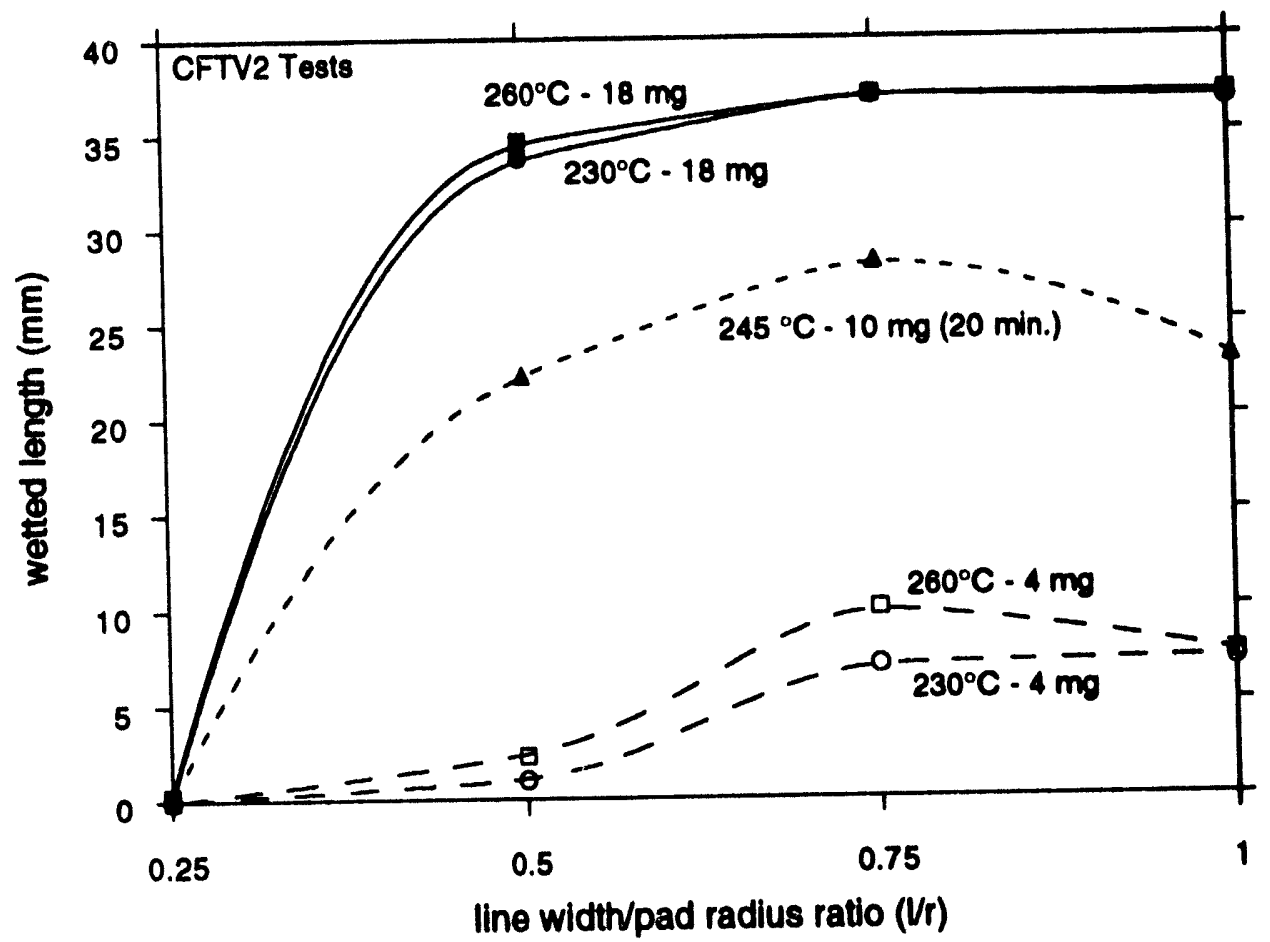

Figure 9. Nominal CFTV2 wetted length as a function of different line widths (pad radius $\left.=0.040^{\prime \prime}\right)$, solder pellet weights, and test temperatures with a 30 minute flux predry (twenty minute predry for the $245^{\circ} \mathrm{C}$ tests). 
External Distribution:

1 Dr. J. Lee Parker AT\&T 4500 Laburnum Avenue Richmond, VA 23231

1 Mr. George Wenger

AT\&T-ERC

P.O. Box 900

Princeton, NJ 08540-0900

1 Dr. Bob Opila

AT\&T

Bell Laboratories 600 Mountain Avenue Murray Hill, NJ 07974

$1 \quad$ Dr. Ed Fey

IBM

1701 North Street

Bldg. 257-2

Endicott, NY 13760

1 Mr. Jim Reed

Texas Instruments

P.O. Box 149149

Austin, TX 78714-9149

$1 \quad$ Alan Burkett

Texas Instruments

2501 S. Highway 121

P.O. Box 4053464

Lewisville, TX 75067

1 Mr. Charles DeSantis

United Technologies Corporation

Hamilton Standard Division

One Hamilton Road

Windsor Locks, CT 06096-1010

1 Mr. Jay Kokas

United Technologies Corporation

Hamilton Standard Division

One Hamilton Road

Windsor Locks, CT 06096-1010

$25 \quad$ Mr. Ron Evans

NCMS

3025 Boardwalk

Ann Arbor, MI 48108-1779

1 Ms. Tracy Pattok

NCMS

3025 Boardwalk

Ann Arbor, MI 48108-1779

1 Dr. John R. Manning

NIST

Materials Bldg. A 153

Gaithersburg, MD 20899

Internal Distribution:

1 MS 0161 M. Moss, 11510

10957 G. L. Cessac, 2411

10336 A. K. Hays, 1709

10337 A. D. Romig, 1800

$10340 \quad$ J. L. Jellison, 1800A

10340 M. Essien, 1831

250340 C. L. Hernandez, 1831

10340 E. A. Holm, 1831

$25 \quad 0340$ F. M. Hosking, 1831

10340 D. O. MacCallum, 1831

$1 \quad 0340 \quad$ J. A. Rejent, 1831

$10340 \quad$ P. T. Vianco, 1831

$25 \quad 0340 \quad$ S. J. Sackinger, 1831

250340 F. G. Yost, 1831

10340 R. Salzbrenner, 1832

10340 D. R. Frear, 1832

$10340 \quad$ N. R. Sorensen, 1832

10340 M. J. Cieslak, 1831

10367 C. L. Renschler, 1812

10367 D. E. Peebles, 1812

10368 H. C. Peebles, 1815

19018 Central Tech. Files, 8523-2

50899 Technical Library, 7141

10619 Tech. Publications, 7151

100100 Document Processing for DOE/OSTI, 7613-2 

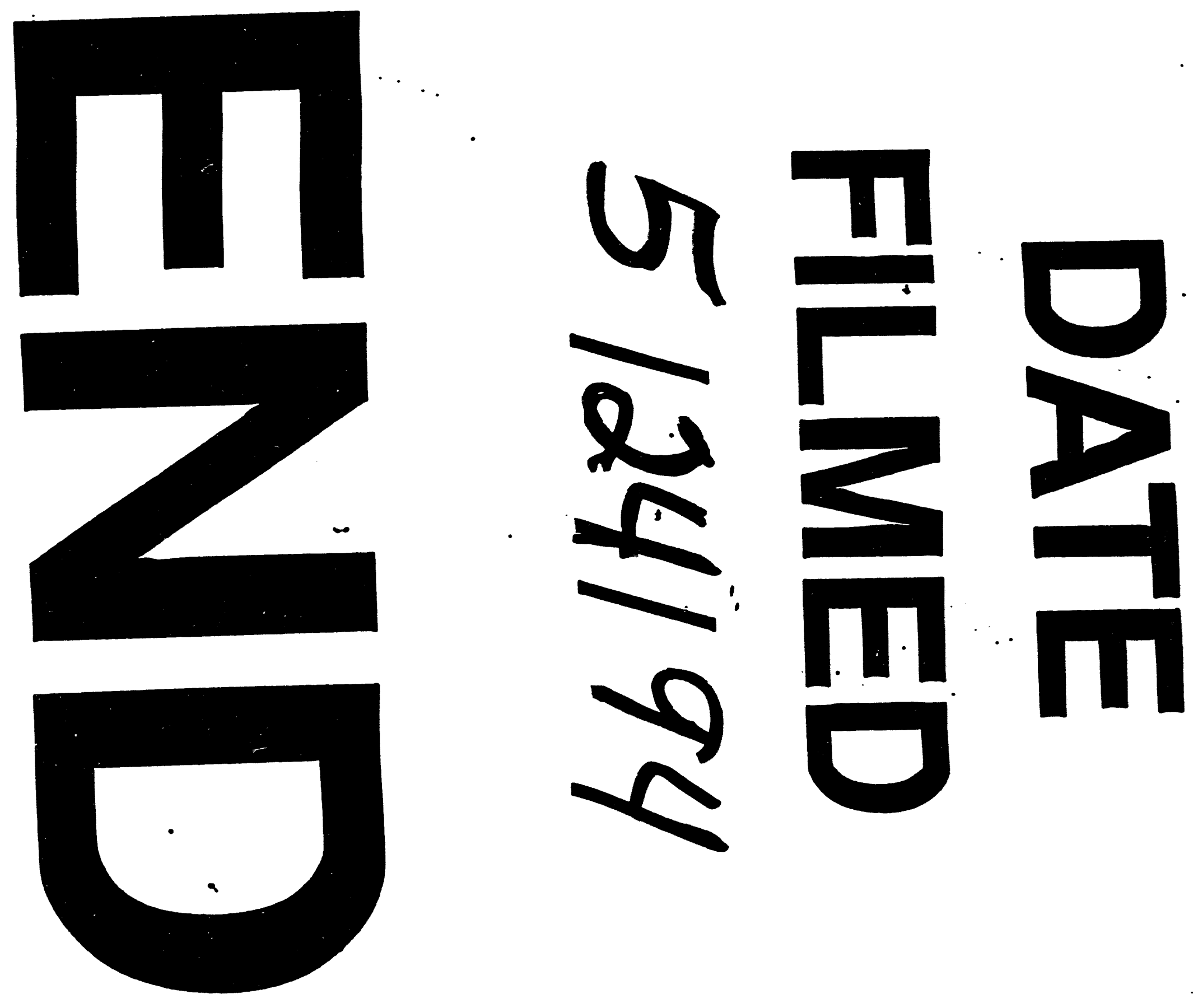


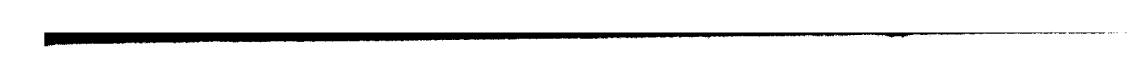

$$
-
$$

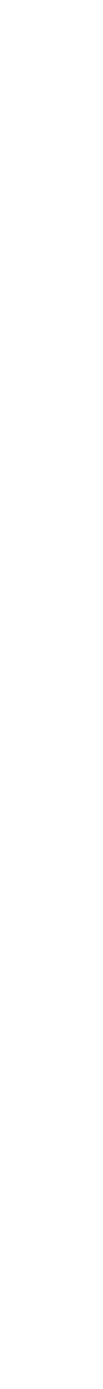

